\title{
RIS-Assisted Space-Air-Ground Integrated Networks: New Horizons for Flexible Access and Connectivity
}

\author{
Lina Bariah, Lina Mohjazi, Hanaa Abumarshoud, Bassant Selim, Sami Muhaidat, \\ Mallik Tatipamula, Muhammad Ali Imran, and Harald Haas
}

\begin{abstract}
Space-air-ground integrated network (SAGIN) has emerged as a paradigm shifting architecture that offers largescale, flexible wireless coverage and seamless, high-rate connectivity to complement terrestrial communications. Nonetheless, unlocking the potentials of SAGIN is subject to addressing nontrivial challenges associated with their inherent time-variability, self-organization, and heterogeneity. Meanwhile, the concept of reconfigurable intelligent surfaces (RISs) is recognized as a disruptive technology that supports an unprecedented control of electromagnetic waves propagation and potentially offers significant enhancements in spectral efficiency, coverage expansion, and security, all achieved in a low-cost and energy-efficient manner. We anticipate that the integration of RISs into SAGIN will not only play a fundamental role in improving the quality of interand intra- layer communications, but will also provide complex interactions among the three network segments, and hence, opens the horizons for a new breed of applications across all industries. In this article, we explore the underlying opportunities and challenges of integrating RIS-enabled communications into SAGIN, and present a forward-looking overview of the crosslayer operational elements supported by RISs. Finally, we outline major enabling technologies and present a look ahead toward addressing open research issues.
\end{abstract}

\section{INTRODUCTION}

Although fifth generation (5G) wireless systems exhibit remarkable performance enhancements compared to their predecessors, both the number of connected devices and the demand for high data rate services to support new technological trends are growing at a phenomenal rate, pushing the current terrestrial telecommunications infrastructure to its limits [1]. Driven by this, the conceptualization of beyond $5 \mathrm{G}$ (B5G) wireless systems, including the sixth generation (6G), is conceived as a set of breakthrough technologies aiming to deliver ultra-high connection reliability, ultra-low latency, and extremely high data rates that support the huge data influx of emerging autonomous and user-centric services.

Supporting truly seamless connectivity and ubiquitous coverage, which are the backbone of the grand $\mathrm{B} 5 \mathrm{G}$ and $6 \mathrm{G}$ visions, calls for a radical departure from conventional wireless communications that solely rely on ground communication systems, which are limited in network capacity, coverage, and deployment cost. Space-air-ground integrated networks (SAGINs) have emerged as a paradigm shift for complementing ground-level communications with space and aerial communications [2], and thereby, offer several advantages in terms of high throughput, large coverage, and strong resilience. However, despite the promising aspects that inter-layer communications can offer, the inherent characteristics of SAGIN, including time-variability, self-organization, and heterogeneity, introduce nontrivial challenges for network design and opera- tion that need to be addressed before the practical roll-out of such networks.

Recently, there has been an upsurge of interest in the development of reconfigurable intelligent surfaces (RISs), thanks to their considerable potential in boosting the spectrum and energy efficiency of wireless networks in a cost-effective manner [3]. A RIS comprises a large number of passive reflective elements (REs), each is an artificial structure that may be proactively and individually reconfigured to achieve a desired overall electromagnetic (EM) response for the surface. As a result, this disruptive technology is capable of transforming the propagation of EM waves into a fully controllable entity. Advances in modern electronics, such as recent improvements in micro-electromechanical systems, make it possible to dynamically manipulate the parameters of the impinging waves (amplitude, phase shift, etc.), allowing for unprecedented EM properties, such as negative refraction and customized permittivity and permeability [1]. This opens the horizons for unique EM waves interactions and functionalities, which include wave focusing, absorption, imaging, scattering, and polarization. It is worth mentioning that RISs support these unique abilities without any limitation on their operating frequency.

The opportunities introduced by RISs and their flexible deployment, low-power use, reduced cost, and lightweight render them as an attractive candidate for supporting SAGIN communications and network integration [4], [5]. It is envisioned that RISs will play a pivotal role in meeting the ambitious requirements of SAGIN, as illustrated in Fig. 1. Recently, a few research papers focused on investigating the potentials of RIS-assisted communications in either single-layer nonterrestrial networks, such as aerial [4] or space [6] networks, or two-layer networks, such as aerial-ground networks [5]. However, to unlock the full potentials of RIS-assisted SAGIN, it is essential to address the challenges associated with the RIS deployment across the whole SAGIN.

Motivated by this, and unlike [4], [6], in this article we divulge the role that RISs can play to address the key challenges associated with SAGIN. To the best of our knowledge, this is the first time in the open literature where an overview and a vision forward is offered on the various facets of the integration of RISs in cross-layer operation in a holistic fashion. Fig. 2 presents the main points discussed in this article.

\section{SAGIN ARCHITECTURE AND RIS INTEGRATION}

Here, we give an overview of the physical layer aspects intertwined with each layer of SAGIN, and shed light on the 


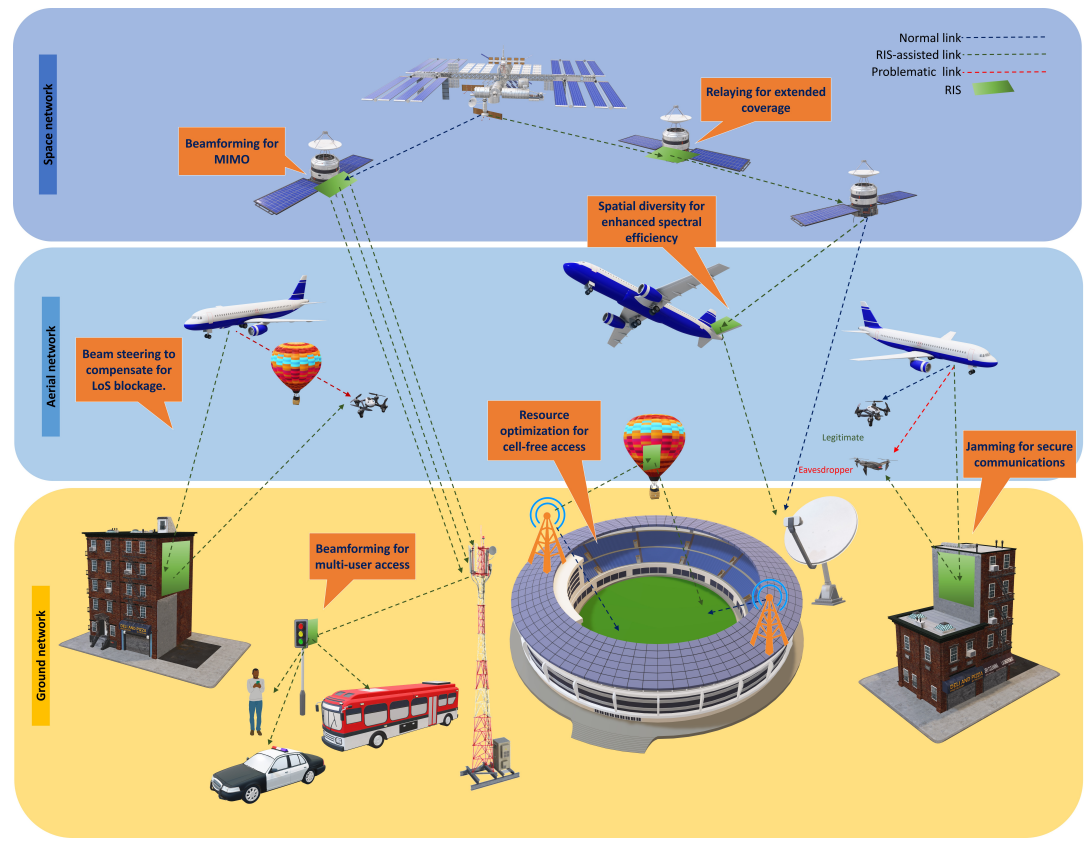

Fig. 1. An architecture for RIS-assisted SAGIN.

opportunities and challenges related to RIS integration.

\section{A. RIS-Assisted Satellite Communications}

The satellite network (SN) comprises satellites and constellations along with their associated ground stations and control centers. Due to the inherently extended coverage and high throughput, satellite communication is deemed a main player in integrated networks as it enables service access in areas with limited or no terrestrial coverage, while providing a reliable backbone alternative to optical fiber backhaul networks.

Future satellite communications are envisioned to adopt two main trends, namely: moving to higher frequency bands and mass-scale deployment. The first trend is driven by spectrum scarcity and is promoting satellite operators to utilize new spectra, which offers much greater signal bandwidth albeit being more susceptible to adverse weather conditions. Higher frequencies enable the use of smaller antenna sizes, thus promoting the integration of multiple-input multiple-output (MIMO) satellite transceivers. Moreover, at such high frequencies, the narrow beams and high directivity necessitates perfect line-of-sight (LoS) alignment between the transmitter and receiver antennas, which needs to be ensured by tracking and stabilization mechanisms [6]. The second trend is manifested through employing swarms of small-power small-size satellites to achieve ubiquitous and flexible connectivity [7]. Low earth orbit (LEO) satellites are particularly suited for such deployments due to their low production and deployment costs.

It is noted that the simultaneous adoption of the two trends will be a challenge; since the realization of multi-beam transmissions with LoS links, at high frequencies, requires the deployment of a large number of antennas and active relaying elements. This contradicts with the power limitation imposed by mass-scale connectivity. Subsequently, RISs, which mainly consist of passive components, present unique prospects for enhancing satellite communication links in an energy-efficient manner.

1) Opportunities:

- Energy Efficient Relaying: The limited on-board processing capabilities of satellites constitute a major obstacle in the implementation of satellite relay networks, particularly between satellites belonging to different orbital planes and/or aerial platforms and ground nodes. To this end, a passive RIS mounted on a satellite can effectively resolve such issue, where it can be fine-tuned so as to enable the REs to act as passive reflectors between the respective layers and, therefore, maintain low energy consumption and limited processing overhead.

- Enhanced Link Reliability: Large path loss and severe signal attenuation represent critical limiting factors in satellite communications. RISs can be optimized in order to ensure maximized signal quality, and therefore, enhanced satellite communication within a limited power budget. In addition to optimizing the RIS configuration, employing a larger number of REs can have a considerable effect on the received signal strength, and hence, can guarantee reliable signal reception and detection.

\section{2) Challenges:}

- Operation and Maintenance: Given the high manufacturing and launching costs, satellites rely on reliable and long-operational lifetime hardware to guarantee a minimum satellite failure-rate. The majority of satellites orbiting in space remain functioning in their missions for several years. However, the operational lifetime and the maintenance frequency of RISs are yet to be thoroughly studied and tested in order to avoid satellite failure which causes interrupted service and financial losses. 


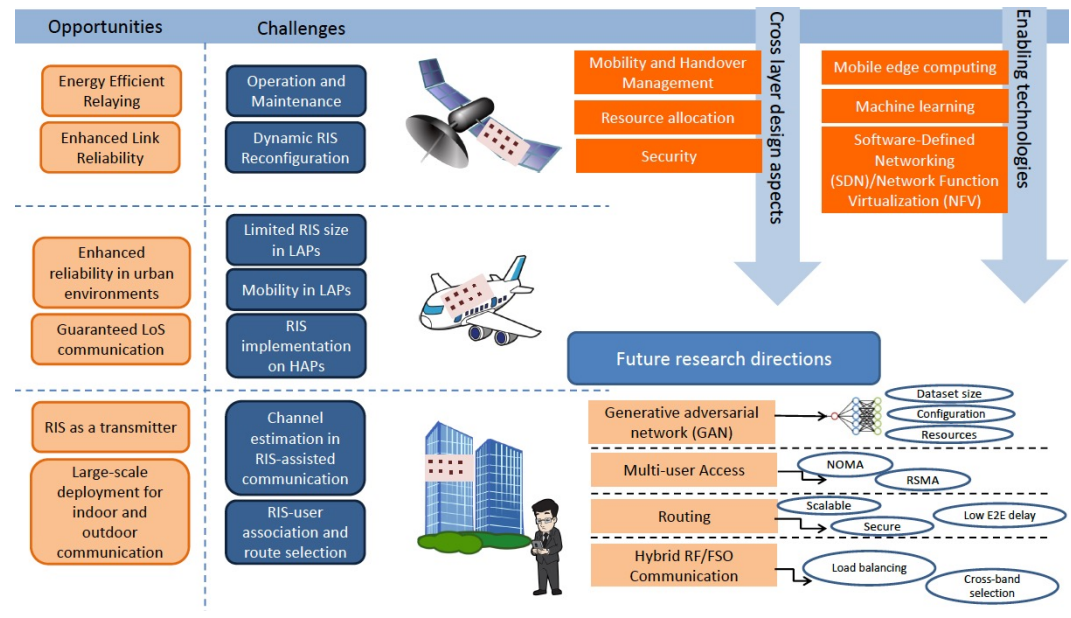

Fig. 2. RIS-enabled SAGIN: Opportunities, design aspects, enabling technologies, challenges, and future directions.

- Dynamic RIS Reconfiguration: RIS reconfiguration overhead represents another major issue facing the integration of RISs in SNs. Particularly, a considerable number of LEO satellites change their orbital planes due to emergency situations [7]. In such scenarios, a RISequipped satellite should adapt its orbital plane to fit the new network configuration and, therefore, a sophisticated level of coordination between satellites is required in order to ensure optimum replacement and reconfiguration of RIS-equipped satellites.

\section{B. RIS-Assisted Aerial Communications}

Aerial communications utilize low altitude platforms (LAPs), e.g. balloons and unmanned aerial vehicles (UAVs), and high altitude platforms (HAPs), e.g. airplanes, as transmitters/receivers for wireless connectivity. Aerial networks (ANs) typically utilize licensed radio frequency (RF) spectrum, allowing service providers to offer guaranteed interference-free and secure connectivity ranging from low-data rate telematics to high bandwidth video streaming. By moving to the unlicensed spectrum, e.g., optical bands, even higher data rates can be achieved, but this requires perfect alignment for LoS connectivity.

The main feature of aerial nodes is their 3D mobility and flexible altitude, which allow them to provide adaptive backhaul links for on-demand terrestrial communications, especially in emergency situations or in the absence of network infrastructure. They also support front-haul aerial connectivity for air traffic management and flight control, and act as an interface between ground and SNs. The agility of ANs constitutes an appealing feature that promotes the concept of integrating RISs, in which a $360^{\circ}$ panoramic full-angle reflection can be realized. Owing to the different shapes and altitudes of aerial platforms, RISs can be integrated in several ways; from the altitude perspective, RISs can be mounted horizontally at the top or the bottom of aerial platforms so as to enable enhanced LAPs-HAPs wireless communication. Alternatively, if the shape of the aerial platform allows, RISs can be implemented as a coating layers on the sides of aerial platforms. The RIS configuration in an aerial platform can be managed by an on-board control unit, which receives optimized operational parameters from either a ground basestation or a flying base-station, and configures the RIS' elements accordingly.

1) Opportunities:

- Enhanced Reliability in Urban Environments: Signal transmission in harsh environments is susceptible to several reflections and scattering, which result in severe signal attenuation in ANs. Therefore, the beamforming and reflection functionalities of RISs, mounted on aerial platforms, can be leveraged in order to manipulate received multi-path signals in urban areas and reflect the desired signal into the intended receiver, achieving a reduced power loss and enhanced transmission/reception reliability.

- Guaranteed LoS Communication: Establishing a LoS link between a UAV and other nodes, including ground, aerial, or satellite nodes, is essential for the successful deployment of ANs. However, several factors can affect the availability of the LoS component, including shadowing and blockage. Hence, the panoramic full-angle reflection, that can be achieved when integrating RISs with aerial platforms, constitutes a promising solution to a such challenge. In particular, in addition to the inherently introduced elevation by aerial platforms, the incorporation of RISs improves the probability of LoS availability.

2) Challenges:

- Limited RIS Size in LAPs: Compared to LAPs, RIS implemented on a HAP can accommodate a larger number of REs, due to the increased length of HAPs with respect to LAPs, where the length of the former ranges from few meters to $200 \mathrm{~m}$ [4]. This results in enhanced signal-tonoise ratio and hence, improved signal quality can be achieved in HAPs. On the contrary, in an attempt to guarantee flight flexibility and stability, several constraints are imposed on the size of RIS implemented on LAPs, particularly in the presence of turbulence.

- Mobility in LAPs: HAPs hover at relatively lower mobility than LAPs and are, therefore, considered quasi- 
stationary platforms. This implies that channel estimation overhead, and subsequently RIS reconfiguration, is reduced in HAPs. On the other hand, increased mobility in LAPs requires frequent channel state information (CSI) acquisition and, hence, continuous RIS reconfiguration.

- RIS Implementation on HAPs: The curved shapes of HAPs introduce different kinds of challenges for the implementation of RIS in aerial platforms. In particular, in curved RIS, the angles of arrival and departure of each RE are different. Hence, attaining the optimum configuration of the RIS brings up additional critical design aspects to consider. Meanwhile, a flat RIS can be easily mounted at the top or the bottom of a UAV.

\section{RIS-Assisted Ground Communications}

Assisted by other network layers, terrestrial communications will remain the main solution to satisfy the majority of the data traffic demands. The ground $6 \mathrm{G}$ network will be characterized by ultra-dense deployment and diverse communication scenarios, hence, in order to facilitate the cross-layer operation between terrestrial and non-terrestrial networks, a versatile and flexible control architecture is needed to effectively make centralized and/or distributed decisions on network operation and service provisioning.

RISs have introduced several promising features to ground wireless communications, including improved quality-ofservice (QoS), enhanced energy and spectral efficiency, lower latency, full-band response, adaptability, reconfigurability, and extended coverage [3]. Besides playing a role in enhancing outdoor wireless links, RISs have also shown potential for enhancing indoor communications, where they can be mounted inside the buildings to strengthen/null/direct signals coming from the cellular network or to optimize the last-mile connectivity, e.g., in WiFi and $\mathrm{LiFi}$ networks [8].

1) Opportunities:

- RIS as a Transmitter: Passive RISs were initially proposed as an effective alternative to active relays and passive non-reconfigurable surfaces. However, recent testbed results corroborated the efficiency of RISs as transmitters. In particular, it was demonstrated that by employing a nearby ground RF source and a particular number of REs, in addition to manipulating the varactor diodes bias voltage, transmitters with various modulation schemes and orders can be realized at the RIS [3]. Such advancements in transmitter designs constitute a transformative step in the field of wireless communications, enabling a wide range of energy, size, and cost-limited applications.

- Large-Scale Deployment for Indoor and Outdoor Communications: Unlike SNs and ANs, ground communications can be enabled by a larger number of RISs, due to the loosened constraints of RIS size and weight at the ground. RISs can be implemented in different sizes on building facades, vehicles, advertising signs, and traffic signals in order to enable reliable outdoor wireless communications, for different networks topologies. Furthermore, NTT DOCOMO, INC. has recently announced the successful deployment of a transparent ultra-thin RIS [9], which can be mounted on windows, rendering it an efficient interface to support indoor-outdoor high frequency wireless communications.

\section{2) Challenges:}

- Channel Estimation in RIS Initiated Communications: The absence of power amplifiers and channel estimation elements in RIS-initiated communications give rise to new design issues, which necessitate the development of novel channel estimation algorithms to acquire the required information, needed for the control and configuration of REs.

- RIS-User Association and Route Selection: Large-scale connectivity in multi-RIS networks calls for developing efficient RIS-user association algorithms, that assign users into corresponding RISs, in order to achieve desired system requirements. For example, an optimum route between ground and aerial or satellite nodes can be selected based on: i) the availability of a LoS link through multiple RISs, ii) the shortest transmitter-RISs-receiver distance, iii) maximized link security, or iv) minimum number of hops.

\section{RIS FOR ENHANCED CROSS-LAYER OPERATION}

Next, we discuss some of the most critical aspects of network integration, highlighting the role that RISs play in ensuring seamless and effective operations.

\section{A. Mobility and Handover Management}

The dynamic topology of integrated networks introduce great diversity in the coverage areas, altitudes, transmission speeds, and mobility patterns among the different serving entities, posing a real challenge when performing mobility management. This is particularly pronounced in vertical handover, since the transceivers' front-ends in this case have distinct mobility patterns. Such handovers can be necessary when, for example, a user enters an area with no ground/air coverage and the connectivity has to be ensured by satellites. Therefore, it is widely acknowledged that efficient intersegment handover mechanisms need to be devised in order to ensure the seamless transition between the space-air-ground segments. However, the need to coordinate frequent handovers, both in horizontal and vertical manners, will result in a heavy mobility management load and large handover delays. RISs offer the flexibility to extend the coverage to beyond the LoS coverage region which, when combined with advanced machine learning (ML)-based mobility prediction algorithms, can ease the burden of mobility overhead by reducing pingpong handovers [10].

\section{B. Resource Allocation}

The heterogeneous nature of SAGIN induces several challenges for QoS provisioning and resource optimization, which is mainly attributed to the imbalance between the resources available at different segments. Hence, RISs can be used to program the wireless environment, thus disrupting the concept of fixed cell boundaries defined by the path loss and the transmission power in legacy networks. RISs can be configured to work as relays and extend the coverage to poorly served cells. On the other hand, the beamforming and reflection functionalities of RISs mounted parallel to the target user 
can be used to mitigate the interference that can arise if point-to-point communications are carried out with closely located users. Such functionalities enhance the signal quality and coverage area of SAGIN and, therefore, ease the job of network self-organizing.

\section{Physical Layer Security}

SAGIN are expected to support numerous military and mission-critical applications, which impose stringent requirements in terms of link reliability and security. Meanwhile, the open nature of wireless communications, the heterogeneity of the communication technologies and infrastructures, the dynamic network configuration, and the pronounced mobility, complicate the task of providing efficient security mechanisms that can resist malicious attacks, such as jamming, eavesdropping, message tampering, etc. Here, from a physical layer security perspective, the benefits of RISs are two-fold. First, following the same approach described for interference management, RISs can be used to reflect the transmitted signal towards the legitimate user, thus alleviating the eavesdropping attacks threat. Second, by appropriately adjusting the reflected signal's amplitude and phase, RISs can be used to cancel the signal at the users' or attacker's side, thus overcoming the aforementioned threats.

\section{EnABling TECHNOLOGIES}

This section presents key enabling technologies that are envisioned to pave the way for the next-generation integrated networks, while highlighting how they shake hands with RISs.

\section{A. Mobile edge computing (MEC)}

MEC exploits computing and storage resources placed at network edges to alleviate the traffic congestion and reduce the response time, especially for delay-sensitive computing tasks. SAGIN are flagged as the next frontier for relieving conventional terrestrial MEC from becoming overwhelmed by the vast number of connection requests and computing demands [11]. Through SAGIN, the systematic and complementary integration of computing, communication, and storage resources from space, air, and ground segments can be actively explored to offer efficient resource utilization and on-demand deployment densification of edge facilities, in addition to ubiquitous, broadband, and reliable computing services. Contrary to conventional MEC-enhanced SAGIN where the wireless propagation environment is uncontrollable, we envision that the integration of RISs will provide these systems with an extra degree of freedom by deliberately controlling the channel conditions to enhance the performance of MEC-enabled SAGIN significantly. By jointly optimizing the communications and computing resources with the RIS coefficients design, the offloading links in the uplink and/or downlink may be dramatically enhanced. This yields the overall energy efficiency of the network to be improved and/or the execution latency to be reduced notably [12]. For example, the amplitudes and phase shifts of the signals reflected from RISs can be proactively adjusted to establish virtual LoS links or enhance the channel gains of the offloading links, in order to enhance the offloading rate and, consequently, immensely reduce the offloading delay.

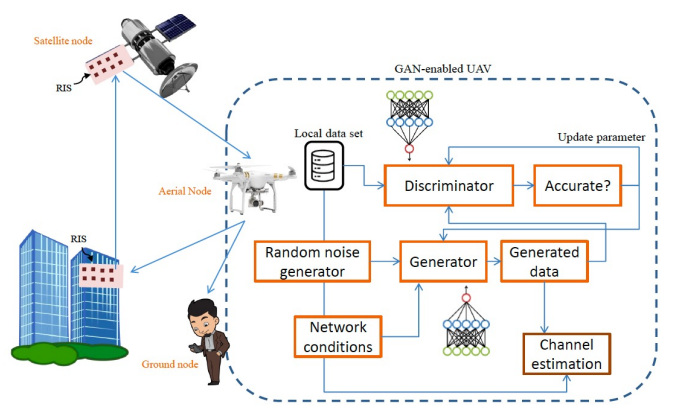

Fig. 3. GAN in RIS-assisted SAGIN for accurate channel estimation.

\section{B. Machine Learning}

SAGINs are expected to support several mission-critical applications, such as disaster relief and military applications, and, therefore, need to operate in a proactive manner to satisfy the corresponding latency and reliability constraints. Hence, although RIS-aided SAGINs exhibit enhanced network reliability, it will impose an increased complexity on the network operation and optimization tasks. To address these concerns, ML is expected to be embedded in the fabric of next generation RIS-assisted SAGINs [13], rather than being a means to solve performance bottlenecks, as is currently the case with $5 \mathrm{G}$. Combined with the improved computational and storage capabilities offered by space, air, and ground nodes, and the collaborative capabilities of the cloudification of the radio access network, ML will enable self-optimizing, self-organizing, and self-healing networks. In the context of RIS-enabled SAGINs, ML can be used to optimize the cell/user/RIS association, and allow for proactive resource management. Despite their promising advantages, classical centralized ML algorithms face three main challenges, namely, compromised data privacy, long propagation delay, and increased network overhead. In this regard, federated learning (FL) was recently identified as a prominent paradigm for provisioning decentralized network optimization and reconfiguration, by enabling low-latency and secure training with reduced network overhead, compared to centralized ML algorithms. Note that, in FL, end-devices (clients) leverage their on-board capabilities with their local datasets in order to train local models, which are then shared with a cloud-based server for aggregation and global model evaluation. Hence, FL is a natural choice for RIS-empowered SAGIN, in which clients are selected from different layers, and accordingly, a network-wide training through all vertical layers promises to deliver a generalized global model that fits all heterogeneous nodes/layers and fulfills their diverse QoS requirements.

\section{Software-Defined Networking (SDN)/Network Function Virtualization (NFV)}

The heterogeneity nature of SAGINs and the extra overhead introduced by RIS control and optimization call for novel techniques to form a unified interface, that configures and controls such network architectures, as well as, manages the network communication protocols [14]. Particularly, SDN through NFV can be a highly promising approach to orchestrate interand intra- operations of heterogeneous nodes over different 
layers in SAGINs. NFV enables offloading the communication protocols management overhead to a virtual platform. By datacontrol decoupling and high-level resource abstraction, SDNenabled nodes merely follow the instructions received from the controller. We anticipate that SDN/NFV will be a key enabler for controlling and optimizing the operations of RIS-assisted SAGIN, since it is expected that communications among the unit elements of RIS are handled by a nano-communication network [3]. In this vision, virtual networks (VNs) are created through NFV to deliver diverse services requested by different SDN-enabled SAGIN nodes, in addition to realizing fully adaptive RIS. Consequently, multiple functionalities can be concurrently performed at each RIS in SAGIN to achieve a particular EM behavior. Hence, according to the varying services requirements, the $\mathrm{VN}$ can be reconfigured to adapt the overall network topology and communication protocols to fit the demands of the new services. This provisions fast network reconfigurability, flexible resource allocation, and guaranteed end-to-end (E2E) QoS.

\section{Collaborative Blockchain}

In the context of SAGIN, blockchain can offer an effective security mechanism allowing diverse entities across the different segments to interact with each other in a verifiable manner using cryptography. Specifically, decentralized control can be performed for the configuration, registration, and verification of inter- and intra-layer operations without the need for a central authority to dictate the rules, resulting in enhanced reliability, intelligence, and transparency compared to traditional techniques. The large-scale operation, highspeed mobility, and low latency requirements of SAGIN bring distinct challenges when it comes to the use of blockchain methods, mainly due to the high overhead of storing replicas at each node as well as the latency caused by the sequential validation of requests. One approach to facilitate a more efficient operation is the simultaneous execution of multiple parallel blockchains, one for securing each network segment, as well as a collaborative blockchain for handling inter-segment cooperation. Albeit offering higher efficiency and lower latency, this approach necessitates sophisticated blockchain collaboration and resource management. In this context, RIS-assisted SAGIN can benefit from dedicating some of the RIS operations to manage the resource interaction and information authentication in each blockchain, i.e., space chain, air chain, and ground chain, in an independent manner, as well as facilitating the interoperability of these blockchains through cross-layer RIS-assisted links.

\section{OPEN ISSUES AND RESEARCH DIRECTIONS}

\section{A. Generative Adversarial Networks for SAGIN}

E2E channel estimation represents a major issue in the implementation of RIS-empowered SAGIN due to diverse mobility patterns experienced by different nodes, different propagation environments between multiple SAGIN layers, and distinct noise types and impairments experienced at each segment. ML algorithms offer a solution for obtaining accurate channel estimation in a centralized or distributed manner.
Centralized ML faces several issues related to complexity, high power requirements, and data privacy, while distributed ML relies on the available data-sets at each device to perform local channel estimation, which may be insufficient to capture all extreme environment scenarios and, hence, requires frequent channel estimation.

Generative adversarial network (GAN) is deemed as a promising solution for efficient distributed channel estimation. In particular, GAN is utilized to generate close-to-real data that captures channels' behaviors and create new data-sets samples representing all channels conditions (Fig. 3) [15]. Despite its promising advantages, GAN-enabled channel estimation in RIS-assisted SAGIN represents an open research issue. Specifically, the size of data-sets required for accurate samples generation, the applicability of GANs in SAGIN, the effect of RIS size and configuration, and the required resources for the implementation of GAN in SAGIN are yet to be thoroughly investigated.

\section{B. Multi-User Access}

The integration of multiple network layers offers a solid support for multi-user access, due to the improved coverage experienced in integrated networks. This can be reinforced by the implementation of RIS-enabled SAGIN, in which multiuser interference can be controlled by the proper configuration of the RIS parameters. Several multiple access schemes can be integrated with RIS-enabled SAGIN in order to support massive-scale connectivity and introduce enhanced energy efficiency, achievable rate, and QoS. This includes non-orthogonal multiple access (NOMA) and rate-splitting multiple access (RSMA) schemes. However, resource allocation, security, and effects of channel/hardware impairments are yet to be investigated when employing NOMA/RSMA in the context of RIS-empowered SAGIN, particularly when these schemes are applied vertically through multiple heterogeneous layers.

\section{Routing}

The design of efficient routing schemes is essential in SAGIN in order to satisfy the diverse QoS requirements of different nodes in different layers. In such dynamic and heterogeneous networks, any changes in the network settings directly impact higher-layers decisions of routing and, therefore, affects their latency, throughput, and reliability performance. With the integration of multiple RISs, the design of efficient routing protocols becomes more challenging due to the increased complexity associated with the RIS configuration and optimization needed to design scalable and secure data routing frameworks that enjoy minimum path-loss, interference, and delay. Despite its important role, routing protocol design in RIS-enabled SAGIN is not investigated in the literature yet, rendering it an attractive open research direction.

\section{Hybrid RF/FSO Communication for SAGIN}

Compared to RF links, free-space optical (FSO) communication offers higher data rates, reduced power consumption, enhanced link security, and higher degrees of spatial diversity. Nonetheless, FSO communication is typically limited by the adverse effects of beam wander and beam scintillation, 
caused by atmospheric inhomogeneities, and the pointing errors resulting from transmitter-receiver misalignment. Thus, employing a hybrid network consisting of an FSO link and a back up RF link is a viable solution to mitigate the downsides of FSO. In such configurations, RIS can be utilized to equip the hybrid links with the agility and adaptability needed to maintain a reliable connection subject to turbulence effects and link disruption. It is noted that the performance analysis of hybrid RF/FSO communication is mostly limited to terrestrial communication in the open literature. Hence, there is a need to model and analyze RIS-assisted hybrid RF/FSO SAGIN links and evaluate the load balancing and cross-band selection combining techniques needed to facilitate high link reliability and comparable data rates.

\section{CONCLUSION}

In this article, we laid down a forward-looking vision for empowering SAGIN with the RIS paradigm. We overviewed the opportunities and challenges introduced by this integration, considering the distinctive natures of different network layers' architecture. We discussed how the merits of RIS can be leveraged to address the ramifications resulting from the crosslayer operation of this platform. We outlined key technologies that would enable a broader manifestation of RIS-assisted SAGIN. Finally, we highlighted several issues that require a thorough investigation to understand the capabilities and limitations of this disruptive innovation before its real-world realization in future.

\section{REFERENCES}

[1] L. Bariah et al., "A prospective look: Key enabling technologies, applications and open research topics in $6 \mathrm{G}$ networks," IEEE Access, vol. 8, pp. 174792-174820, Aug. 2020.

[2] J. Liu, Y. Shi, Z. M. Fadlullah, and N. Kato, "Space-air-ground integrated network: A survey," IEEE Commun. Surveys Tuts., vol. 20, no. 4, pp. 2714-2741, May 2018.

[3] M. Di Renzo et al., "Smart radio environments empowered by reconfigurable intelligent surfaces: How it works, state of research, and the road ahead," IEEE J. Sel. Areas Commun., vol. 38, no. 11, pp. 2450-2525, Nov. 2020.

[4] S. Alfattani et al., "Aerial platforms with reconfigurable smart surfaces for 5G and beyond," IEEE Commun. Mag., vol. 59, no. 1, pp. 96-102, Jan. 2021.

[5] S. Li et al., "Reconfigurable intelligent surface assisted UAV communication: Joint trajectory design and passive beamforming," IEEE Wireless Commun. Lett., vol. 9, no. 5, pp. 716-720, May 2020.

[6] K. Tekbıyık, G. K. Kurt, A. R. Ekti, A. Görçin, and H. Yanikomeroglu, "Reconfigurable intelligent surfaces empowered $\mathrm{THz}$ communication in LEO satellite networks," arXiv preprint arXiv:2007.04281v3, Apr. 2021.

[7] S. Xu, X.-W. Wang, and M. Huang, "Software-defined next-generation satellite networks: Architecture, challenges, and solutions," IEEE Access, vol. 6, pp. 4027-4041, Feb. 2018.

[8] H. Abumarshoud et al., "LiFi through reconfigurable intelligent surfaces: A new frontier for 6G?" arXiv preprint arXiv:2104.02390v3, Apr. 2021.

[9] "DOCOMO and AGC use metasurface lens to enhance radio signal reception indoors - new technology efficiently guides millimeter waves to target locations indoors," Jan. 2021.

[10] N. Abuzainab, M. Alrabeiah, A. Alkhateeb, and Y. E. Sagduyu, "Deep learning for $\mathrm{THz}$ drones with flying intelligent surfaces: Beam and handoff prediction," arXiv preprint arXiv:2102.11222v1, Feb. 2021.

[11] S. Yu et al., "EC-SAGINs: Edge computing-enhanced space-air-ground integrated networks for internet of vehicles," IEEE Internet Things J., pp. 1-1, Jan. 2021.

[12] T. Bai, C. Pan, C. Han, and L. Hanzo, "Empowering mobile edge computing by exploiting reconfigurable intelligent surface," arXiv preprint arXiv:2102.02569, Feb. 2021
[13] L. Mohjazi et al., "An outlook on the interplay of artificial intelligence and software-defined metasurfaces: An overview of opportunities and limitations," IEEE Veh. Technol. Mag., vol. 15, no. 4, pp. 62-73, Dec. 2020.

[14] Z. Zhou et al., "SAGECELL: Software-defined space-air-ground integrated moving cells," IEEE Commun. Mag., vol. 56, no. 8, pp. 92-99, Aug. 2018.

[15] A. Ferdowsi and W. Saad, "Brainstorming generative adversarial networks (BGANs): Towards multi-agent generative models with distributed private datasets," arXiv preprint arXiv:2002.00306, 2020.

\section{BIOGRAPHIES}

Lina Bariah (lina.bariah@ieee.org) received the Ph.D. degree in communications engineering from Khalifa University, UAE, in 2018. She is a Postdoctoral Fellow with the KU Center for Cyber-Physical Systems, Khalifa University, and an affiliate research fellow, School of Engineering, University of Glasgow, UK.

Lina Mohjazi (Lina.Mohjazi@glasgow.ac.uk) is a Lecturer in the School of Engineering, University of Glasgow, UK. She received her Ph.D. degree from the University of Surrey, UK, in 2018. She is Associate Editor for IEEE Communications Letters.

Hanaa Abumarshoud (hanaa.abumarshoud@strath.ac.uk) is a Research Associate at the University of Strathclyde, UK. She was awarded her MSc and PhD in Electrical and Computer Engineering in 2013 and 2017 from Khalifa University, UAE.

Bassant Selim (bassant.selim@ericsson.com) received the Ph.D. degree from Khalifa University, UAE, in 2017. She is a Data Scientist with Ericsson's Global AI Accelerator, Montréal, QC, Canada.

Sami Muhaidat (muhaidat@ieee.org) received the Ph.D. degree in electrical and computer engineering from the University of Waterloo, Waterloo, in 2006. He is a Professor with Khalifa University and an Adjunct Professor with Carleton University, Canada.

Mallik Tatipamula (mallik.tatipamula@ericsson.com) is a CTO at Ericsson, leading evolution of Ericsson's technology, and championing the company's next phase of innovation and growth driven by $5 \mathrm{G}$ distributed multi-cloud deployments. $\mathrm{He}$ also leads O-RAN and $6 \mathrm{G}$ research efforts.

Muhammad Ali Imran (Muhammad.Imran@glasgow.ac.uk) is a Professor of communication systems with the University of Glasgow, UK, and Dean of Glasgow College-UESTC. He is an Affiliate Professor with University of Oklahoma, USA, and a Visiting Professor at University of Surrey, UK.

Harald Haas (harald.haas@strath.ac.uk) is the Director of the LiFi Research and Development Centre, University of Strathclyde, UK. He is also Co-Founder and Chief Scientific Officer of pureLiFi Ltd. 